\title{
EXACT BOUNDARY CONTROLLABILITY OF A HYBRID SYSTEM OF ELASTICITY BY THE HUM METHOD
}

\author{
BOPENG RAO ${ }^{1}$
}

\begin{abstract}
We consider the exact controllability of a hybrid system consisting of an elastic beam, clamped at one end and attached at the other end to a rigid antenna. Such a system is governed by one partial differential equation and two ordinary differential equations. Using the HUM method, we prove that the hybrid system is exactly controllable in an arbitrarily short time in the usual energy space.
\end{abstract}

Mathematics Subject Classification. 93C20, 35B37, 35D05, 73K50.

Received July 14, 1999. Revised August 31 and November 22, 2000.

\section{INTRODUCTION}

In this work we consider a hybrid system consisting of an elastic beam of length $L$, clamped at one end and attached at the other end to a rigid antenna, whereon are applied the dynamical controls $v_{0}, v_{1}$. The vibration $y(x, t)$ of the beam is governed by the Euler-Bernoulli equation, and the oscillations $y(L, t), y_{x}(L, t)$ of the antenna are described by the Newton-Euler equations through which the control dynamics are filtered:

$$
\begin{cases}y_{t t}+y_{x x x x}=0 & 0<x<L, \quad t>0, \\ y(0, t)=y_{x}(0, t)=0 & t>0, \\ \rho y_{t t}(L, t)-y_{x x x}(L, t)=v_{0}(t) & t>0, \\ J y_{x t t}(L, t)+y_{x x}(L, t)=v_{1}(t) & t>0, \\ y(x, 0)=y_{0}(x), \quad y_{t}(x, 0)=y_{1}(x) & 0<x<L\end{cases}
$$

where $\rho>0$ is the mass and $J>0$ is the moment of inertia of the antenna. For more details concerning the descriptions of the physical structure of the system, we refer to Littman and Markus [3].

For smooth initial data: $y_{0} \in H^{6}(0, L), y_{1} \in H^{4}(0, L)$, the exact controllability of the system (1.1) was demonstrated by Littman and Markus [3]. Their method is based on the theory of semi-infinite beam. The

Keywords and phrases: Hybrid system, weak solution, exact controllability, singular control, unique continuation.

${ }^{1}$ Institut de Recherche Mathématique Avancée, Université Louis Pasteur de Strasbourg, 7 rue René-Descartes, 67084 Strasbourg

Cedex, France; e-mail: rao@math.u-strasbg.fr 
existence of the smooth open-loop controllers $v_{0}, v_{1}$ for the finite beam system (1.1) follows from a constructive cut-off approach.

But in the case of usual initial data: $y_{0} \in H^{2}(0, L), y_{1} \in L^{2}(0, L)$, the regularity of weak solution is insufficient to define the traces $y_{t t}(1, t)$ and $y_{x t t}(1, t)$. In that case following an idea of Slemrod [15], the boundary conditions involving the dynamical terms $y_{t t}(1, t)$ and $y_{x t t}(1, t)$ can be treated as two ordinary differential equations (with respect to the time variable $t)$. More specifically, denoting by $u(x, t)=\left(y(x, t), y(L, t), y_{x}(L, t)\right)$ the state of the system (1.1) and by $v=\left(0, v_{0}, v_{1}\right)$ the control, we transform the system (1.1) into an abstract system

$$
u_{t t}+A u=v, \quad u(0)=u_{0}, \quad u_{t}(0)=u_{1}
$$

where $A$ is a positive definite operator in the product space $L^{2}(0, L) \times \mathbf{R}^{2}$. We obtain thus a weak formulation of the original system (1.1). Although the system (1.2) is well posed in the sense of semi-groups of contractions, the regularity of the weak solution is insufficient to define the traces of derivatives of higher order $y_{t t}(1, t)$ and $y_{x t t}(1, t)$. Accordingly, the exact controllability of the hybrid system would appear to be impossible for usual initial data (see [3], p. 223).

We know that the closed-loop system with the usual boundary feedback $v_{1}=y_{t}(1, t), \quad v_{2}=y_{x t}(1, t)$ is not uniformly stabilizable (see $[4,12]$ ), because the usual feedback is compact in the corresponding energy space. But this incomplete result gave a false impression that the Hilbert uniqueness method (Lions [5] and [6]) couldn't be adapted to such problems (see [7], p. 984). In fact, we established in [12] the uniform stability of the hybrid system (1.1) by means of boundary feedback of higher order such that $v_{0}=y_{x x x t}(1, t), \quad v_{1}=-y_{x x t}(1, t)$ which is necessarily non-compact. This indicates that the system (1.1) could be exactly controllable by means of singular controllers $v_{0}, v_{1}$.

Other hybrid systems were studied in $[1,2]$ and [8]. It was observed that the solutions of hybrid systems gain one more degree regularity when crossing the point mass. This property allows to define the trace at the point mass. However the controllable initial data is not the whole usual space in which the system was well-posed. It was also remarked that the hybrid systems have non spectral cap condition and the observability inequalities were established by means of non harmonic Fourier's series, such as inequality of Ingham or Ulrich. In all these works, the exact controllability was only proved for the initial data which have one more degree regularity.

In this work (the results of which were announced in Rao [13]), we will adapt the Hilbert uniqueness method to the exact controllability of the abstract problem (1.2). The main difficulty in this approach consists in establishing an inverse energy inequality, the direct energy inequality is easy to establish for this kind of problem. Inspired from the uniform stability results, we look for the estimates of the traces of higher order of the solution of the associated homogeneous problem. This allows us to establish the exact controllability of the abstract system (1.2) for usual initial data by means of two singular controllers $v_{0}, v_{1}$.

Another interesting problem is to know whether the abstract problem (1.2) is exactly controllable using only one control $v_{1}$. We recall that this problem was open even in the case of smooth initial data. Here guided by a uniqueness result [10], we consider a beam of limited length $L<3$. We prove that the system (1.2) is exactly controllable by only one controller $v_{1}$ in an arbitrarily short time for usual initial data.

The paper is organized as follows: in Section 2 we consider the associated homogeneous problem. The direct and inverse energy inequalities are established with the usual norm. In Section 3 we consider the exact controllability of the abstract problem (1.1) with usual initial data. We first prove the abstract system (1.2) is exactly controllable in an arbitrarily short time by two controls $v_{0} \in L^{2}(0, T), v_{1} \in\left(H^{1}(0, T)\right)^{\prime}$ for usual initial data $y_{0} \in H^{2}(0, L), y_{1} \in L^{2}(0, L)$ with compatibility conditions. Next in the case of a beam of limited length $L<3$, we establish also the exact controllability of the system(1.1) by means of only one singular controller $\left(H^{1}(0, T)\right)^{\prime}$. In Section 4, extend the exact controllability results to the case of more regular initial data $y_{0} \in H^{4}(0, L), y_{1} \in H^{2}(0, L)$ with compatibility conditions. We will prove that the abstract system (1.2) is exactly controllable in an arbitrarily short time by means of two controls $v_{0} \in H^{1}(0, T), v_{1} \in L^{2}(0, L)$, or by means of only one controller $v_{1} \in L^{2}(0, L)$ for a beam of limited length $L<3$. 


\section{Homogeneous System}

In this section we consider the homogeneous problem:

$$
\begin{array}{lc}
\phi_{t t}+\phi_{x x x x}=0 & t>0, \quad 0<x<L, \\
\phi(0, t)=\phi_{x}(0, t)=0 & t>0, \\
\phi_{t t}(L, t)-\phi_{x x x}(L, t)=0 & t>0, \\
\phi_{x t t}(L, t)+\phi_{x x}(L, t)=0 & t>0 .
\end{array}
$$

Since the physical constants $\rho, J$ are strictly positive, without loss of generality, we will take $\rho=J=1$ throughout this paper. We first write formally the system (2.1-2.4) into

$$
\left(\phi(x, t), \phi(L, t), \phi_{x}(L, t)\right)_{t t}+\left(\phi_{x x x x}(x, t),-\phi_{x x x}(L, t), \phi_{x x}(L, t)\right)=0 .
$$

According to this formulation, we introduce the product space

$$
H=L^{2}(0, L) \times \mathbf{R} \times \mathbf{R}
$$

endowed with the usual norm

$$
\|\Phi\|_{H}^{2}=\int_{0}^{L} \phi^{2} d x+\xi^{2}+\eta^{2}, \quad \forall \Phi=(\phi, \xi, \eta) \in H
$$

We next define the linear operator $A$ as follows

$$
\begin{gathered}
D(A)=\left(\begin{array}{c}
\Phi=(\phi, \xi, \eta): \\
\phi \in H^{4}(0, L) \\
\phi(0)=\phi_{x}(0)=0 ; \quad \xi=\phi(L), \eta=\phi_{x}(L)
\end{array}\right), \\
A \Phi=\left(\phi_{x x x x},-\phi_{x x x}(L), \phi_{x x}(L)\right), \quad \forall \Phi=(\phi, \xi, \eta) \in D(A) .
\end{gathered}
$$

Then setting

$$
\xi(t)=\phi(L, t), \quad \eta(t)=\phi_{x}(L, t), \quad \Phi(x, t)=(\phi(x, t), \xi(t), \eta(t))
$$

we write the equation (2.5) into an evolutionary equation

$$
\Phi_{t t}+A \Phi=0, \quad \Phi(0)=\Phi_{0}, \quad \Phi_{t}(0)=\Phi_{1} .
$$

Proposition 2.1. The operator $A$ defined in $(2.8,2.9)$ is self-adjoint and definite positive. Moreover $A^{-1}$ is compact in $H$.

Proof. We first prove that $A$ is a symmetric operator in $H$. A straightforward computation gives that

$$
(A \Phi, \widetilde{\Phi})_{H}=\int_{0}^{L} \phi_{x x} \widetilde{\phi}_{x x} d x=(\Phi, A \widetilde{\Phi})_{H}
$$

for any $\Phi=(\phi, \xi, \eta), \widetilde{\Phi}=(\widetilde{\phi}, \widetilde{\xi}, \widetilde{\eta}) \in D(A)$. In particular, using Poincaré's inequality we get

$$
(A \Phi, \Phi)_{H}=\int_{0}^{L} \phi_{x x}^{2} d x \geq C\left\{\int_{0}^{L} \phi^{2} d x+\phi^{2}(L)+\phi_{x}^{2}(L)\right\}=C\|\Phi\|_{H}^{2} .
$$


Now let $\widetilde{\Phi}=(\widetilde{\phi}, \widetilde{\xi}, \widetilde{\eta}) \in D\left(A^{*}\right)$, then there exists $\Phi_{0}=\left(\phi_{0}, \xi_{0}, \eta_{0}\right) \in H$ such that $(\widetilde{\Phi}, A \Phi)=\left(\Phi_{0}, \Phi\right)$ for all $\Phi \in D(A)$. This means that

$$
\int_{0}^{L} \widetilde{\phi} \phi_{x x x x} d x-\widetilde{\xi} \phi_{x x x}(L)+\widetilde{\eta} \phi_{x x}(L)=\int_{0}^{L} \phi_{0} \phi d x+\xi_{0} \phi(L)+\eta_{0} \phi_{x}(L)
$$

for all $\phi \in H^{4}(0,1)$ satisfying $\phi(0)=\phi_{x}(0)=0$. Then indeed a straightforward computation shows that

$$
\widetilde{\phi} \in H^{4}(0, L): \quad \widetilde{\phi}(0)=\widetilde{\phi}_{x}(0)=0, \quad \widetilde{\xi}=\widetilde{\phi}(L), \quad \widetilde{\eta}=\widetilde{\phi}_{x}(L) .
$$

It follows that $\widetilde{\Phi} \in D(A)$, therefore we obtain $A^{*}=A$.

Finally let $\Phi=\left(\phi, \phi(L), \phi_{x}(L)\right) \in D(A)$ solve the equation $A \Phi=\Phi_{0}$ for $\Phi_{0}=\left(\phi_{0}, \xi_{0}, \eta_{0}\right) \in H$. Then we have

$$
\left\{\begin{array}{l}
\phi_{x x x x}=\phi_{0} \quad 0<x<L, \\
\phi(0)=\phi_{x}(0)=0, \\
\phi_{x x}(L)=\eta_{0}, \quad \phi_{x x x}(L)=-\xi_{0} .
\end{array}\right.
$$

There exists a unique solution $\phi \in H^{4}(0, L)$ such that $\|\phi\|_{H^{4}(0, L)} \leq C\left\|\Phi_{0}\right\|_{H}$. We obtain thus the compactness of $A^{-1}$. The proof is complete.

Since $A$ is self-adjoint and definite positive, and since $A^{-1}$ is compact, using the spectral decomposition theory, we can define the powers $A^{2 \alpha} \in \mathcal{L}\left(D\left(A^{\alpha}\right) ; D\left(A^{-\alpha}\right)\right)$ for any $\alpha \in \mathbf{R}$, where the domain $D\left(A^{\alpha}\right)$ is endowed with the norm

$$
\|\Phi\|_{D\left(A^{\alpha}\right)}^{2}=\left\|A^{\alpha} \Phi\right\|_{H}^{2}, \quad \forall \Phi \in D\left(A^{\alpha}\right) .
$$

In particular, we have

$$
\begin{gathered}
D\left(A^{1 / 2}\right)=\left(\begin{array}{c}
\Phi=(\phi, \xi, \eta) \in H: \phi \in H^{2}(0, L) \\
\phi(0)=\phi_{x}(0)=0 ; \quad \xi=\phi(L), \eta=\phi_{x}(L)
\end{array}\right), \\
\|\Phi\|_{V}^{2}=\int_{0}^{L} \phi_{x x}^{2} d x, \quad \forall \Phi=(\phi, \xi, \eta) \in V .
\end{gathered}
$$

For convenience, in the following we write $V=D\left(A^{1 / 2}\right)$.

The following result is classic (see [9]).

Proposition 2.2. Assume that $\left(\Phi_{0}, \Phi_{1}\right) \in D\left(A^{\alpha+1 / 2}\right) \times D\left(A^{\alpha}\right)$. Then the equation (2.11) admits a unique solution $\Phi(t)$ such that

$$
\Phi(t) \in C^{0}\left(\mathbf{R}, D\left(A^{\alpha+1 / 2}\right) \cap C^{1}\left(\mathbf{R}, D\left(A^{\alpha}\right)\right) .\right.
$$

Moreover for any $t \in \mathbf{R}$ :

$$
\|\Phi(t)\|_{D\left(A^{\alpha+1 / 2}\right)}^{2}+\left\|\Phi_{t}(t)\right\|_{D\left(A^{\alpha}\right)}^{2}=\left\|\Phi_{0}\right\|_{D\left(A^{\alpha+1 / 2}\right)}^{2}+\left\|\Phi_{1}\right\|_{D\left(A^{\alpha}\right)}^{2} .
$$

Theorem 2.3. Let $\Phi(x, t)=\left(\phi(x, t), \phi(L, t), \phi_{x}(L, t)\right)$ be the solution of the equation (2.11). Then for any $T>0$, there exist constants $C_{1}>0$ and $C_{2}>0$ depending only on $T$ such that the following estimates hold

$$
C_{1} \int_{0}^{T}\left(\left|\phi_{t}(L, t)-\beta \phi_{t}(L, 0)\right|^{2}+\phi_{x t t}^{2}(L, t)\right) d t \leq\left\|\Phi_{0}\right\|_{V}^{2}+\left\|\Phi_{1}\right\|_{H}^{2},
$$




$$
\left\|\Phi_{0}\right\|_{V}^{2}+\left\|\Phi_{1}\right\|_{H}^{2} \leq C_{2} \int_{0}^{T}\left(\left|\phi_{t}(L, t)-\beta \phi_{t}(L, 0)\right|^{2}+\phi_{x t t}^{2}(L, t)\right) d t
$$

where $\beta$ is an arbitrary real number that will be chosen later $(\beta=0$ in Th. 3.1 and $\beta=1$ in Th. 4.1).

Proof. Since $D\left(A^{\infty}\right) \times D\left(A^{\infty}\right)$ is dense in $V \times H$, we can assume that $\phi$ is sufficiently smooth. Then indeed, we have

$$
\left\|\Phi_{t}(t)\right\|_{H}^{2}=\int_{0}^{L} \phi_{t}^{2}(x, t) d x+\phi_{t}^{2}(L, t)+\phi_{x t}^{2}(L, t) .
$$

Therefore using (2.16) and (2.19), it is easy to verify that the direct inequality (2.17) is equivalent to the following one

$$
C_{1} \int_{0}^{T} \phi_{x t t}^{2}(L, t) d t \leq\left\|\Phi_{0}\right\|_{V}^{2}+\left\|\Phi_{1}\right\|_{H}^{2}
$$

Now multiplying the equation $(2.1)$ by $2 x \phi_{x}$ and integrating by parts, we obtain that

$$
\begin{aligned}
\int_{0}^{L} \int_{0}^{T}\left(\phi_{t}^{2}(x, t)+3 \phi_{x x}^{2}(x, t)\right) d x d t= & -2 \int_{0}^{L}\left[\phi_{t} x \phi_{x}\right]_{0}^{T} d x+L \int_{0}^{T}\left(\phi_{t}^{2}(L, t)+\phi_{x x}^{2}(L, t)\right) d t \\
& +2 \int_{0}^{T}\left(\phi_{x x}(L, t)-L \phi_{x x x}(L, t)\right) \phi_{x}(L, t) d t .
\end{aligned}
$$

Using the boundary conditions (2.3)-(2.4), it follows that

$$
\begin{aligned}
\int_{0}^{T}\left(\phi_{x x}(L, t)-L \phi_{x x x}(L, t)\right) \phi_{x}(L, t) d t= & -\int_{0}^{T}\left(\phi_{x t t}(L, t)+L \phi_{t t}(L, t)\right) \phi_{x}(L, t) d t \\
= & -\left[\left(\phi_{x t}(L, t)+L \phi_{t}(L, t)\right) \phi_{x}(L, t)\right]_{0}^{T} \\
& +\int_{0}^{T}\left(\phi_{x t}(L, t)+L \phi_{t}(L, t)\right) \phi_{x t}(L, t) d t
\end{aligned}
$$

Inserting (2.22) into (2.21) and noting (2.4), we obtain

$$
\begin{aligned}
L \int_{0}^{T}\left(\phi_{t}^{2}(L, t)+\phi_{x t t}^{2}(L, t)\right) d t= & \int_{0}^{T} \int_{0}^{L}\left(\phi_{t}(x, t)^{2}+3 \phi_{x x}^{2}(x, t)\right) d x d t-2 \int_{0}^{T}\left(L \phi_{t}(L, t) \phi_{x t}(L, t)+\phi_{x t}^{2}(L, t)\right) d t \\
& +2 \int_{0}^{L}\left[\phi_{t} x \phi_{x}\right]_{0}^{T} d x+2\left[\left(\phi_{x t}(L, t)+L \phi_{t}(L, t)\right) \phi_{x}(L, t)\right]_{0}^{T} .
\end{aligned}
$$

Using Cauchy-Schwartz inequality in (2.23), we deduce easily the direct inequality (2.20). We next write (2.23) into

$$
\begin{aligned}
\int_{0}^{T} \int_{0}^{L}\left(\phi_{t}(x, t)^{2}+3 \phi_{x x}^{2}(x, t)\right) d x d t & +\int_{0}^{T}\left(\phi_{t}^{2}(L, t)+\phi_{x t}^{2}(L, t)\right) d t=\int_{0}^{T}\left((L+1) \phi_{t}^{2}(L, t)+L \phi_{x t t}^{2}(L, t)\right) d t \\
& +\int_{0}^{T}\left(3 \phi_{x t}^{2}(L, t)+2 L \phi_{t}(L, t) \phi_{x t}(L, t)\right) d t \\
& -2 \int_{0}^{L}\left[\phi_{t} x \phi_{x}\right]_{0}^{T} d x-2\left[\left(\phi_{x t}(L, t)+L \phi_{t}(L, t)\right) \phi_{x}(L, t)\right]_{0}^{T} . \quad(2.24)
\end{aligned}
$$


On the other hand, a direct computation gives

$$
\begin{aligned}
\int_{0}^{T}\left(3 \phi_{x t}^{2}(L, t)+2 L \phi_{t}(L, t) \phi_{x t}(L, t)\right) d t= & {\left[\left(2 L \phi(L, t)+3 \phi_{x}(L, t)\right) \phi_{x t}(L, t)\right]_{0}^{T} } \\
& -\int_{0}^{T}\left(3 \phi_{x}(L, t)+2 L \phi(L, t)\right) \phi_{x t t}(L, t) d t .
\end{aligned}
$$

Inserting (2.25) into (2.24) gives

$$
\begin{aligned}
\int_{0}^{T} \int_{0}^{L}\left(\phi_{t}^{2}(x, t)+3 \phi_{x x}^{2}(x, t)\right) d x d t & +\int_{0}^{T}\left(\phi_{t}^{2}(L, t)+\phi_{x t}^{2}(L, t)\right) d t=\int_{0}^{T}\left((L+1) \phi_{t}^{2}(L, t)+L \phi_{x t t}^{2}(L, t)\right) d t \\
& -\int_{0}^{T}\left(3 \phi_{x}(L, t)+2 L \phi(L, t)\right) \phi_{x t t}(L, t) d t-2 \int_{0}^{L}\left[\phi_{t} x \phi_{x}\right]_{0}^{T} d x \\
& +\left[\left(2 L \phi(L, t)+\phi_{x}(L, t)\right) \phi_{x t}(L, t)-2 L \phi_{x}(L, t) \phi_{t}(L, t)\right]_{0}^{T} .
\end{aligned}
$$

Now applying Cauchy-Schwartz inequality, we obtain that

$$
\begin{gathered}
\left|\int_{0}^{T}\left(3 \phi_{x}(L, t)+2 L \phi(L, t)\right) \phi_{x t t}(L, t) d t\right| \leq \int_{0}^{T} \phi_{x t t}^{2}(L, t) d t+C\|\phi\|_{L^{\infty}\left(0, T ; H^{s}(0, L)\right)}^{2} . \\
\left|\int_{0}^{L} \phi_{t} x \phi_{x} d x\right| \leq \epsilon\left\|\Phi_{t}(t)\right\|_{H}^{2}+C_{\epsilon}\|\phi\|_{L^{\infty}\left(0, T ; H^{s}(0, L)\right)}^{2}, \\
\left|\left(2 L \phi(L, t)+\phi_{x}(L, t)\right) \phi_{x t}(L, t)-2 L \phi_{t}(L, t) \phi_{x}(L, t)\right| \leq \epsilon\left\|\Phi_{t}(t)\right\|_{H}^{2}+C_{\epsilon}\|\phi\|_{L^{\infty}\left(0, T ; H^{s}(0, L)\right)}^{2}
\end{gathered}
$$

provided $3 / 2<s<2$.

Finally inserting (2.27-2.29) into (2.26) gives that

$$
\|\Phi(t)\|_{V}^{2}+\left\|\Phi_{t}(t)\right\|_{H}^{2} \leq C\left(\int_{0}^{T}\left(\phi_{t}^{2}(L, t)+\phi_{x t t}^{2}(L, t)\right) d t+\|\phi\|_{L^{\infty}\left(0, T ; H^{s}(0, L)\right)}^{2}\right)
$$

provided $0<6 \epsilon<T$ and $3 / 2<s<2$.

We will use a compactness-uniqueness argument of Zuazua [16] to prove that the term of lower order $\|\phi\|_{L^{\infty}\left(0, T ; H^{s}(0, L)\right)}^{2}$ can be absorbed. Assume that (2.18) fails, then there exists a sequence $\left(\Phi_{0}^{n}, \Phi_{1}^{n}\right) \in D\left(A^{\infty}\right)$ $\times D\left(A^{\infty}\right)$ such that

$$
\begin{gathered}
\left\|\Phi^{n}(t)\right\|_{V}^{2}+\left\|\Phi_{t}^{n}(t)\right\|_{H}^{2}=1, \quad \forall t \in \mathbf{R}, \\
\int_{0}^{T}\left(\left|\phi_{t}^{n}(L, t)-\beta \phi_{t}^{n}(L, 0)\right|^{2}+\left|\phi_{x t t}^{n}(L, t)\right|^{2}\right) d t \rightarrow 0
\end{gathered}
$$

where $\Phi^{n}(x, t)=\left(\phi^{n}(x, t), \phi^{n}(L, t), \phi_{x}^{n}(L, t)\right)$ is the solution of the following equation

$$
\Phi_{t t}^{n}+A \Phi^{n}=0, \quad \Phi^{n}(0)=\Phi_{0}^{n}, \quad \Phi_{t}^{n}(0)=\Phi_{1}^{n} .
$$


Since $\left|\phi_{t}^{n}(L, 0)\right| \leq\left\|\Phi_{1}^{n}\right\|_{H} \leq 1$ therefore there exists a subsequence $\phi_{t}^{n}(L, 0)$, still indexed by $n$ for convenience, that converges to a constant $c$ as $n \rightarrow+\infty$. Hence it follows from (2.32)

$$
\int_{0}^{T}\left(\left|\phi_{t}^{n}(L, t)\right|^{2}+\left|\phi_{x t t}^{n}(L, t)\right|^{2}\right) d t \rightarrow \beta^{2} c^{2} T .
$$

On the other hand, from (2.31) it follows that

$$
\left\|\phi^{n}\right\|_{L^{\infty}\left(0, T ; H^{2}(0, L)\right)}^{2}+\left\|\phi_{t}^{n}\right\|_{L^{\infty}\left(0, T ; L^{2}(0, L)\right)}^{2} \leq 1 .
$$

Thanks to the compact embedding (see Simon [14]), there exists a subsequence $\phi^{n}$, still indexed by $n$ for convenience such that

$$
\phi^{n}(t) \rightarrow \phi(t)
$$

strongly in $L^{\infty}\left(0, T ; H^{s}(0, L)\right)$ for $3 / 2<s<2$. Then using $(2.34)$ and $(2.36)$ in $(2.30)$, we see that $\left(\Phi^{n}(t), \Phi_{t}^{n}(t)\right)$ is a Cauchy sequence in the space $L^{\infty}(0, T ; V \times H)$. This implies that

$$
\left(\Phi^{n}(t), \Phi_{t}^{n}(t)\right) \rightarrow\left(\Phi(t), \Phi_{t}(t)\right)
$$

strongly in $L^{\infty}(0, T ; V \times H)$. Then indeed passing $n \rightarrow \infty$ in $(2.31-2.33)$, we deduce that $\Phi(x, t)=(\phi(x, t), \phi(L, t)$, $\left.\phi_{x}(L, t)\right)$ solves the equation

$$
\Phi_{t t}(t)+A \Phi(t)=0, \quad \Phi(0)=\Phi_{0}, \quad \Phi_{t}(0)=\Phi_{1},
$$

and satisfies the following supplementary conditions

$$
\begin{gathered}
\phi_{t}(L, t)=\beta c, \quad \phi_{x t t}(L, t)=0, \\
\|\Phi(t)\|_{V}^{2}+\left\|\Phi_{t}(t)\right\|_{H}^{2}=1, \quad \forall t \in \mathbf{R} .
\end{gathered}
$$

From $(2.37,2.38)$, a straightforward computation gives

$$
\begin{cases}\phi_{t t}+\phi_{x x x x}=0 & t>0, \quad 0<x<L, \\ \phi(0, t)=\phi_{x}(0, t)=0 & t>0, \\ \phi_{t t}(L, t)=\phi_{x x x}(L, t)=0 & t>0 \\ \phi_{x t t}(L, t)=\phi_{x x}(L, t)=0 & t>0 .\end{cases}
$$

Now setting $w=\phi_{t t}$ in (2.40), we find that $w$ solves, in the sense of distributions, the following equation:

$$
\begin{cases}w_{t t}+w_{x x x x}=0 & t>0, \quad 0<x<L \\ w(0, t)=w_{x}(0, t)=0 & t>0, \\ w(L, t)=w_{x}(L, t)=w_{x x}(L, t)=w_{x x x}(L, t)=0, & t>0 .\end{cases}
$$

Applying Holmgren's theorem (Lions [5]), we deduce $\phi_{t t}=0$. This implies in turn that

$$
\begin{cases}\phi_{x x x x}=0 & t>0, \quad 0<x<L, \\ \phi(0, t)=\phi_{x}(0, t)=0 & t>0 \\ \phi_{x x}(L, t)=\phi_{x x x}(L, t)=0 & t>0 .\end{cases}
$$


Multiplying (2.41) by $\phi$ and integrating by parts, we deduce that $\phi \equiv 0$, this contradicts (2.39). The proof is thus complete.

Theorem 2.4. Let $\Phi(x, t)=\left(\phi(x, t), \phi(L, t), \phi_{x}(L, t)\right)$ be the solution of the equation (2.11). Assume that $L<3$. Then for any $T>0$, there exist constants $C_{2}$ depending only on $T, L$ such that the following estimate holds

$$
\left\|\Phi_{0}\right\|_{V}^{2}+\left\|\Phi_{1}\right\|_{H}^{2} \leq C_{2} \int_{0}^{T} \phi_{x t t}^{2}(L, t) d t
$$

Proof. Multiplying the equation (2.1) by $\phi$ and integrating by parts, it follows that

$$
\begin{aligned}
\int_{0}^{T} \int_{0}^{L}\left(\phi_{t}^{2}(x, t)-\phi_{x x}^{2}(x, t)\right) d x d t & +\int_{0}^{T}\left(\phi_{t}^{2}(L, t)+\phi_{x t}^{2}(L, t)\right) d t \\
& =\int_{0}^{L}\left[\phi \phi_{t}\right]_{0}^{T} d x+\left[\phi(L, t) \phi_{t}(L, t)+\phi_{x}(L, t) \phi_{x t}(L, t)\right]_{0}^{T} .
\end{aligned}
$$

Combining (2.26) and (2.43), we get

$$
\begin{aligned}
(1+\delta) \int_{0}^{T} \int_{0}^{L} \phi_{t}^{2}(x, t) d x d t & +(3-\delta) \int_{0}^{T} \int_{0}^{L} \phi_{x x}^{2}(x, t) d x d t+(\delta-L) \int_{0}^{T} \phi_{t}^{2}(L, t) d t+(1+\delta) \int_{0}^{T} \phi_{x t}^{2}(L, t) d t \\
& =\int_{0}^{T}\left(L \phi_{x t t}^{2}(L, t)-\left(3 \phi_{x}(L, t)+2 L \phi(L, t)\right) \phi_{x t t}(L, t)\right) d t \\
& +\int_{0}^{L}\left[(\delta \phi-2 x \phi) \phi_{t}\right]_{0}^{T} d x+\left[\left(2 L \phi(L, t)+(1+\delta) \phi_{x}(L, t)\right) \phi_{x t}(L, t)\right]_{0}^{T} \\
& +\left[\left(\delta \phi(L, t)-2 L \phi_{x}(L, t)\right) \phi_{t}(L, t)\right]_{0}^{T} .
\end{aligned}
$$

Setting $L<\delta<3$ and using the same computation as in (2.27-2.29), we deduce from (2.44) the following estimate

$$
\|\Phi(t)\|_{V}^{2}+\left\|\Phi_{t}(t)\right\|_{H}^{2} \leq C\left(\int_{0}^{T} \phi_{x t t}^{2}(L, t) d t+\|\phi\|_{L^{\infty}\left(0, T ; H^{s}(0, L)\right)}^{2}\right) .
$$

Using the same compactness-uniqueness argument as in Theorem 2.3, we prove easily that the term of lower order $\|\phi\|_{L^{\infty}\left(0, T ; H^{s}(0, L)\right)}^{2}$ can be absorbed. In fact, if (2.42) fails, then the limit function $\phi$ satisfies the following equation:

$$
\begin{cases}\phi_{t t}+\phi_{x x x x}=0 & t>0, \quad 0<x<L, \\ \phi(0, t)=\phi_{x}(0, t)=0 & t>0, \\ \phi_{t t}(L, t)-\phi_{x x x}(L, t)=0 & t>0 \\ \phi_{x t t}(L, t)=\phi_{x x}(L, t)=0 & t>0 .\end{cases}
$$

Following an uniqueness result in Rao [10], we get that $\phi \equiv 0$. The proof is thus complete. 


\section{Exact CONTROLlability FOR USUAL initial DATA}

In this section, we consider the exact controllability of the hybrid system (1.1) with usual initial data. We first give the following direct and inverse inequalities.

Theorem 3.1. Let $\Phi(x, t)=\left(\phi(x, t), \phi(L, t), \phi_{x}(L, t)\right)$ be the solution of the homogeneous equation (2.11). Then for any $T>0$, there exist constants $C_{1}>0$ and $C_{2}>0$ depending only on $T$ such that the following estimates hold

$$
\begin{aligned}
& C_{1} \int_{0}^{T}\left(\phi^{2}(L, t)+\phi_{x t}^{2}(L, t)\right) d t \leq\left\|\Phi_{0}\right\|_{H}^{2}+\left\|\Phi_{1}\right\|_{V^{\prime}}^{2}, \\
& \left\|\Phi_{0}\right\|_{H}^{2}+\left\|\Phi_{1}\right\|_{V^{\prime}}^{2} \leq C_{2} \int_{0}^{T}\left(\phi^{2}(L, t)+\phi_{x t}^{2}(L, t)\right) d t .
\end{aligned}
$$

Proof. Because of the density, we consider only $\left(\Phi_{0}, \Phi_{1}\right) \in D\left(A^{\infty}\right) \times D\left(A^{\infty}\right)$. Defining

$$
\widetilde{\Phi}_{0}=-A^{-1} \Phi_{0}, \quad \widetilde{\Phi}_{1}=\Phi_{0}
$$

we have

$$
\left\|\widetilde{\Phi}_{0}\right\|_{V}^{2}+\left\|\widetilde{\Phi}_{1}\right\|_{H}^{2}=\left\|\Phi_{0}\right\|_{H}^{2}+\left\|\Phi_{1}\right\|_{V^{\prime}}^{2} .
$$

Now let $\widetilde{\Phi}(x, t)=\left(\widetilde{\phi}(x, t), \widetilde{\phi}(L, t), \widetilde{\phi}_{x}(L, t)\right)$ be the solution of the equation

$$
\widetilde{\Phi}_{t t}+A \widetilde{\Phi}=0, \quad \widetilde{\Phi}(0)=\widetilde{\Phi}_{0}, \quad \widetilde{\Phi}_{t}(0)=\widetilde{\Phi}_{1} .
$$

Then applying the inequalities $(2.17,2.18)$ (with $\beta=0)$ to the solution $\widetilde{\Phi}$ of the equation (3.5), it follows that

$$
\begin{gathered}
C_{1} \int_{0}^{T}\left(\widetilde{\phi}_{t}^{2}(L, t)+\widetilde{\phi}_{x t t}^{2}(L, t)\right) d t \leq\left\|\widetilde{\Phi}_{0}\right\|_{V}^{2}+\left\|\widetilde{\Phi}_{1}\right\|_{H}^{2}, \\
\left\|\widetilde{\Phi}_{0}\right\|_{V}^{2}+\left\|\widetilde{\Phi}_{1}\right\|_{H}^{2} \leq C_{2} \int_{0}^{T}\left(\widetilde{\phi}_{t}^{2}(L, t)+\widetilde{\phi}_{x t t}^{2}(L, t)\right) d t .
\end{gathered}
$$

On the other hand, since $\widetilde{\Phi}_{t}(0)=\widetilde{\Phi}_{1}=\Phi_{0}, \quad \widetilde{\Phi}_{t t}(0)=-A \widetilde{\Phi}(0)=\Phi_{1}$, it follows that $\widetilde{\Phi}_{t}=\Phi$. Then replacing $\widetilde{\phi}_{t}$ by $\phi$ in $(3.6,3.7)$ and using $(3.4)$ gives the inequalities $(3.1,3.2)$. The proof is thus complete.

Theorem 3.2. Let $\Phi(x, t)=\left(\phi(x, t), \phi(L, t), \phi_{x}(L, t)\right)$ be the solution of the homogeneous equation (2.11). Assume that $L<3$. Then for any $T>0$, there exist constants $C_{2}>0$ depending only on $T, L$ such that the following estimate holds

$$
\left\|\Phi_{0}\right\|_{H}^{2}+\left\|\Phi_{1}\right\|_{V^{\prime}}^{2} \leq C_{2} \int_{0}^{T} \phi_{x t}^{2}(L, t) d t
$$

Proof. The proof is the same as that of Theorem 3.1. It is sufficient to apply the inverse inequality (2.42) to the solution $\widetilde{\Phi}$ of the equation (3.5). 
Now we consider the following controlled problem

$$
\left\{\begin{array}{l}
y_{t t}+y_{x x x x}=0, \\
y(0, t)=y_{x}(0, t)=0 \\
y_{t t}(L, t)-y_{x x x}(L, t)=v_{0}(t) \\
y_{x t t}(L, t)+y_{x x}(L, t)=v_{1}(t) \\
y(x, 0)=y_{0}(x), \quad y_{t}(x, 0)=y_{1}(x) .
\end{array}\right.
$$

Setting

$$
u(x, t)=\left(y(x, t), y(L, t), y_{x}(L, t)\right), \quad v=\left(0, v_{0}, v_{1}\right)
$$

we write the system (3.9) into the following form:

$$
u_{t t}+A u=v, \quad u(0)=u_{0}, \quad u_{t}(0)=u_{1} .
$$

Let $\Phi$ be a solution of the homogeneous problem (2.11). Multiplying the equation (3.11) by $\Phi$ and integrating by parts so that we obtain formally

$$
\left(u_{0}, \Phi_{1}\right)_{H}-\left(u_{1}, \Phi_{0}\right)_{H}+\int_{0}^{t}\left(v_{0}(s) \phi(L, s)+v_{1}(s) \phi_{x}(L, s)\right) d s=\left(u(t), \Phi_{t}(t)\right)_{H}-\left(u_{t}(t), \Phi(t)\right)_{H} .
$$

Next defining the linear form $L$ by setting

$$
L\left(\Phi_{0}, \Phi_{1}\right)=\left\langle u_{0}, \Phi_{1}\right\rangle_{V \times V^{\prime}}-\left\langle u_{1}, \Phi_{0}\right\rangle_{H \times H}+\int_{0}^{t}\left(v_{0}(s) \phi(L, s)+v_{1}(s) \phi_{x}(L, s)\right) d s
$$

we obtain a weak formulation of the equation (3.11).

$$
L\left(\Phi_{0}, \Phi_{1}\right)=\left\langle\left(-u_{t}(t), u(t)\right), S(t)\left(\Phi_{0}, \Phi_{1}\right)\right\rangle_{H \times V, H \times V^{\prime}}
$$

where $S(t)$ denotes the group of isometrics associated to the homogeneous problem (2.11).

We first consider the exact controllability of the equation (3.11) for usual initial data $\left(\Phi_{0}, \Phi_{1}\right) \in V \times H$. We choose the controls as follows:

$$
v_{0} \in L^{2}(0, T), \quad v_{1}=-\frac{d}{d t} \widetilde{v}_{1} \in\left(H^{1}(0,1)\right)^{\prime}
$$

where the derivative $\frac{d}{d t}$ is defined in the sense of $\left(H^{1}(0,1)\right)^{\prime}$

$$
\int_{0}^{T} \frac{d}{d t} \widetilde{v}_{1}(t) \chi(t) d t=-\int_{0}^{T} \widetilde{v}_{1}(t) \chi_{t}(t) d t, \quad \forall \chi \in H^{1}(0, T) .
$$

Theorem 3.3. For any $\left(u_{0}, u_{1}\right) \in V \times H$ and $\left(v_{0}, v_{1}\right) \in L^{2}(0, T) \times\left(H^{1}(0, T)\right)^{\prime}$, the controlled equation (3.11) admits a unique weak solution $u$ such that

$$
u \in C^{0}([0, T] ; V) \cap C^{1}([0, T] ; H)
$$


defined in the sense that the equation (3.14) is satisfied for all $\left(\Phi_{0}, \Phi_{1}\right) \in H \times V^{\prime}$ and all $0<t<T$. Moreover the linear application

$$
\left(u_{0}, u_{1}, v_{0}, v_{1}\right) \rightarrow\left(u, u_{t}\right)
$$

is continuous for the corresponding topologies.

Proof. First using the definition (3.15) and the direct inequality (3.1), a straightforward computation gives that

$$
\begin{aligned}
\left|\int_{0}^{t}\left(v_{0}(s) \phi(L, s)+v_{1}(s) \phi_{x}(L, s)\right) d s\right|= & \left|\int_{0}^{t}\left(v_{0}(s) \phi(L, s)+\widetilde{v}_{1}(s) \phi_{x t}(L, s)\right) d s\right| \leq C\left(\left\|v_{0}\right\|_{L^{2}(0, T)}\right. \\
& \left.+\left\|\widetilde{v}_{1}\right\|_{L^{2}(0, T)}\right)\left(\|\phi(L, \cdot)\|_{L^{2}(0, T)}+\left\|\phi_{x t}(L, \cdot)\right\|_{L^{2}(0, T)}\right) \\
\leq & C\left(\left\|v_{0}\right\|_{L^{2}(0, T)}+\left\|v_{1}\right\|_{H^{1}(0, T)^{\prime}}\right)\left(\left\|\Phi_{0}\right\|_{H}+\left\|\Phi_{1}\right\|_{V^{\prime}}\right) .
\end{aligned}
$$

This implies that the linear form $L$ is continuous in the space $H \times V^{\prime}$. Moreover we have

$$
\|L\| \leq C\left(\left\|u_{0}\right\|_{V}+\left\|u_{1}\right\|_{H}+\left\|v_{0}\right\|_{L^{2}(0, T)}+\left\|v_{1}\right\|_{H^{1}(0, T)^{\prime}}\right) .
$$

From Riesz's representation theorem, there exists a unique $\left(U(t), U_{t}(t)\right) \in V \times H$ such that

$$
L\left(\Phi_{0}, \Phi_{1}\right)=\left\langle\left(-U_{t}(t), U(t)\right),\left(\Phi_{0}, \Phi_{1}\right)\right\rangle_{H \times V, H \times V^{\prime}}
$$

for all $\left(\Phi_{0}, \Phi_{1}\right) \in H \times V^{\prime}$. Then indeed setting

$$
\left(-u_{t}(t), u(t)\right):=S(t)\left(-U_{t}(t), U(t)\right)
$$

we obtain the equation (3.14). Moreover from (3.19) it follows that

$$
\|u(t)\|_{V}+\left\|u_{t}(t)\right\|_{H} \leq C\left(\left\|u_{0}\right\|_{V}+\left\|u_{1}\right\|_{H}+\left\|v_{0}\right\|_{L^{2}(0, T)}+\left\|v_{1}\right\|_{H^{1}(0, T)^{\prime}}\right) .
$$

Now let $v_{0} \in C^{\infty}([0, T]), v_{1} \in C^{\infty}([0, T])$. We know that the equation (2.11) admits a smooth solution $u$ possessing the regularity (3.17). Since $C^{\infty}([0, T])$ is dense in $L^{2}(0, T)$ and in $H^{1}(0, T)^{\prime}$, by virtue of $(3.20)$ we see that the weak solution $u$ satisfies also the regularity (3.17). The continuous dependence of the application (3.18) follows also from (3.20). The proof is complete.

Theorem 3.4. Let $T>0$. Then for all $\left(u_{0}, u_{1}\right) \in V \times H$, there exist two controllers $v_{0} \in L^{2}(0, T)$ and $v_{1} \in H^{1}(0, T)^{\prime}$ such that the weak solution $u$ of the controlled problem (3.11) satisfies the final conditions

$$
u(T)=u_{t}(T)=0 .
$$

Proof. Let $\Phi$ be the solution of homogeneous system (2.11) with the initial data $\left(\Phi_{0}, \Phi_{1}\right) \in H \times V^{\prime}$. We define the semi-norm

$$
\left\|\left(\Phi_{0}, \Phi_{1}\right)\right\|_{F}^{2}=\int_{0}^{T}\left(\phi^{2}(L, t)+\phi_{x t}^{2}(L, t)\right) d t .
$$

Thanks to the inequalities $(3.1,3.2)$, we know that (3.22) defines an equivalent norm in the space $F=H \times V^{\prime}$. Choosing the controllers $v_{0}, v_{1}$ as follows

$$
v_{0}(t)=\phi(L, t), \quad v_{1}(t)=-\frac{d}{d t} \phi_{x t}(L, t) .
$$


According to the direct inequality (3.1), we have

$$
\left\|v_{0}\right\|_{L^{2}(0, T)}^{2}+\left\|v_{1}\right\|_{H^{1}(0,1)^{\prime}}^{2} \leq C\left(\left\|\Phi_{0}\right\|_{H}^{2}+\left\|\Phi_{1}\right\|_{V^{\prime}}^{2}\right) .
$$

Next we solve the backward problem

$$
u_{t t}+A u=v, \quad u(T)=u_{t}(T)=0 .
$$

Thanks to Theorem 3.2 we see that (3.25) admits a unique weak solution possessing the regularity (3.17). In particular, we have

$$
\|u(t)\|_{V}+\left\|u_{t}(t)\right\|_{H} \leq C\left(\left\|v_{0}\right\|_{L^{2}(0, T)}+\left\|v_{1}\right\|_{H^{1}(0, T)^{\prime}}\right) .
$$

Now defining the operator $\Lambda$ as

$$
\Lambda\left(\Phi_{0}, \Phi_{1}\right)=\left(u_{t}(0),-u(0)\right), \quad \forall\left(\Phi_{0}, \Phi_{1}\right) \in H \times V^{\prime}
$$

by virtue of the inequalities (3.26) and (3.24) we obtain that

$$
\left\|\Lambda\left(\Phi_{0}, \Phi_{1}\right)\right\|_{V \times H} \leq C\left(\left\|v_{0}\right\|_{L^{2}(0, T)}+\left\|v_{1}\right\|_{H^{1}(0, T)^{\prime}}\right) \leq C\left\|\left(\Phi_{0}, \Phi_{1}\right)\right\|_{H \times V^{\prime}} .
$$

This proves that $\Lambda$ is a linear continuous operator from $H \times V^{\prime}$ into $V \times H$.

Now multiplying the backward equation (3.25) by $\Phi$ and integrating by parts so that we obtain that

$$
-\left(u_{0}, \Phi_{1}\right)_{H}+\left(u_{1}, \Phi_{0}\right)_{H}=\int_{0}^{t}\left(\phi^{2}(L, s)+\phi_{x t}^{2}(L, s)\right) d s .
$$

Interpreting (3.28) into the following form

$$
\left\langle\Lambda\left(\Phi_{0}, \Phi_{1}\right),\left(\Phi_{0}, \Phi_{1}\right)\right\rangle_{H \times V, H \times V^{\prime}}=\left\|\left(\Phi_{0}, \Phi_{1}\right)\right\|_{F}^{2}
$$

and using Lax-Milgram's theorem, we deduce that $\Lambda$ is an isomorphism from $H \times V^{\prime}$ onto $H \times V$. Therefore given any $\left(u_{1},-u_{0}\right) \in H \times V$, there exists a unique $\left(\Phi_{0}, \Phi_{1}\right) \in H \times V^{\prime}$ such that

$$
\Lambda\left(\Phi_{0}, \Phi_{1}\right)=\left(u_{t}(0),-u(0)\right)=\left(u_{1},-u_{0}\right) .
$$

This means precisely that the weak solution $u$ of the backward problem (3.25), with the right-hand side $v$ given by (3.23), satisfies the initial value conditions

$$
u(0)=u_{0}, \quad u_{t}(0)=u_{1} .
$$

In other words, we have proved that the system (3.11) is driven to rest by the singular controls $v_{0}, v_{1}$ given in (3.23). The proof is thus complete.

In the following, we consider the exact controllability of (3.11) by means of only one controller. We first give the following inverse inequality.

Theorem 3.5. Let $L<3$ and $T>0$. Then for any $\left(u_{0}, u_{1}\right) \in V \times H$ there exists a control $v_{1} \in H^{1}(0,1)^{\prime}$ such that the solution $u$ of the controlled problem (3.11) satisfies the final conditions (3.21). 
Proof. We first solve the homogeneous system (2.11) for $\left(\Phi_{0}, \Phi_{1}\right) \in H \times V^{\prime}$. Defining the semi-norm

$$
\left\|\left(\Phi_{0}, \Phi_{1}\right)\right\|_{F}^{2}=\int_{0}^{T} \phi_{x t}^{2}(L, t) d t
$$

then thanks to the inequalities (3.1) and (3.8) we know that (3.32) defines an equivalent norm in the space $F=H \times V^{\prime}$.

Choosing the controllers $v_{0}, v_{1}$ as follows

$$
v_{0}=0, \quad v_{1}(t)=-\frac{d}{d t} \phi_{x t}(L, t) .
$$

According to the direct inequality (3.1), we have

$$
\left\|v_{1}\right\|_{H^{1}(0,1)^{\prime}}^{2} \leq C\left(\left\|\Phi_{0}\right\|_{H}^{2}+\left\|\Phi_{1}\right\|_{V^{\prime}}^{2}\right) .
$$

Now we solve again the backward problem (3.25) with the control chosen in (3.33). Recall that in that case Theorem 3.3 remains valid. In particular, we have

$$
\|u(t)\|_{V}+\left\|u_{t}(t)\right\|_{H} \leq C\left\|v_{1}\right\|_{H^{1}(0, T)^{\prime}}
$$

Next we define the operator $\Lambda$ as in (3.25). Then from the inequalities (3.34) and (3.35) we deduce that

$$
\left\|\Lambda\left(\Phi_{0}, \Phi_{1}\right)\right\|_{V \times H} \leq C\left\|v_{1}\right\|_{H^{1}(0, T)^{\prime}} \leq C\left\|\left(\Phi_{0}, \Phi_{1}\right)\right\|_{H \times V^{\prime}} .
$$

Therefore $\Lambda$ is a linear continuous operator from $H \times V^{\prime}$ into $V \times H$.

Now multiplying the equation (3.25) by $\Phi$ and integrating by parts, we obtain that

$$
-\left(u_{0}, \Phi_{1}\right)_{H}+\left(u_{1}, \Phi_{0}\right)_{H}=\int_{0}^{T} \phi_{x t}^{2}(L, s) d s .
$$

Interpreting (3.36) into the following form

$$
\left\langle\Lambda\left(\Phi_{0}, \Phi_{1}\right),\left(\Phi_{0}, \Phi_{1}\right)\right\rangle_{H \times V, H \times V^{\prime}}=\left\|\left(\Phi_{0}, \Phi_{1}\right)\right\|_{F}^{2}
$$

and using Lax-Milgram's theorem we deduce that $\Lambda$ is an isomorphism from $H \times V^{\prime}$ onto $H \times V$. Therefore given any $\left(u_{1},-u_{0}\right) \in H \times V$, there exists a unique $\left(\Phi_{0}, \Phi_{1}\right) \in H \times V^{\prime}$ such that

$$
\Lambda\left(\Phi_{0}, \Phi_{1}\right)=\left(u_{t}(0),-u(0)\right)=\left(u_{1},-u_{0}\right) .
$$

This means precisely that the weak solution $u$ of the backward problem (3.25), with the right-hand side $v$ given by (3.23), satisfies the initial value conditions

$$
u(0)=u_{0}, \quad u_{t}(0)=u_{1} .
$$

In other words, we have proved that the system (3.11) is driven to rest by the singular controls $v_{0}, v_{1}$ given in (3.23). The proof is thus complete. 


\section{Exact CONTROLlability FOR SMOOTh INITIAL DATA}

In the last section, we will consider the exact controllability of the system (3.11) using regular controls. To this end, we will choose a weaker norm $\|\cdot\|_{F}$ (see [5], pp. 122-127). We first give the following estimates.

Theorem 4.1. Let $\Phi(x, t)=\left(\phi(x, t), \phi(L, t), \phi_{x}(L, t)\right)$ be the solution of the equation (2.11). Then for any $T>0$, there exist constants $C_{1}>0$ and $C_{2}>0$ depending only on $T$ such that the following estimates hold

$$
\begin{aligned}
& C_{1} \int_{0}^{T}\left\{\left(\int_{0}^{t} \phi(L, s) d s\right)^{2}+\phi_{x}^{2}(L, t)\right\} d t \leq\left\|\Phi_{0}\right\|_{V^{\prime}}^{2}+\left\|\Phi_{1}\right\|_{D(A)^{\prime}}^{2}, \\
& \left\|\Phi_{0}\right\|_{V^{\prime}}^{2}+\left\|\Phi_{1}\right\|_{D(A)^{\prime}}^{2} \leq C_{2} \int_{0}^{T}\left\{\left(\int_{0}^{t} \phi(L, s) d s\right)^{2}+\phi_{x}^{2}(L, t)\right\} d t .
\end{aligned}
$$

Proof. The proof is similar to that of Theorem 3.1. For the sake of completeness, we give a sketch of the proof. In fact, because of the density we consider only the initial data $\left(\Phi_{0}, \Phi_{1}\right) \in D\left(A^{\infty}\right) \times D\left(A^{\infty}\right)$. Then indeed we define

$$
\widehat{\Phi}_{0}=-A^{-1} \Phi_{0}, \quad \widehat{\Phi}_{1}=-A^{-1} \Phi_{1} .
$$

It is easy to see that

$$
\left\|\widehat{\Phi}_{0}\right\|_{V}^{2}+\left\|\widehat{\Phi}_{1}\right\|_{H}^{2}=\left\|\Phi_{0}\right\|_{V^{\prime}}^{2}+\left\|\Phi_{1}\right\|_{D(A)^{\prime}}^{2}
$$

Let $\widehat{\Phi}$ solve the equation

$$
\widehat{\Phi}_{t t}+A \widehat{\Phi}=0, \quad \widehat{\Phi}(0)=\widehat{\Phi}_{0}, \quad \widehat{\Phi}_{t}(t)=\widehat{\Phi}_{1}(0) .
$$

Applying the inequalities $(2.17,2.18)$ (with $\beta=1$ ) to the solution $\widehat{\Phi}$, we obtain that

$$
\begin{aligned}
& C_{1} \int_{0}^{T}\left(\left|\widehat{\phi}_{t}(L, t)-\widehat{\phi}_{t}(L, 0)\right|^{2}+\widehat{\phi}_{x t t}^{2}(L, t)\right) d t \leq\left\|\widehat{\Phi}_{0}\right\|_{V}^{2}+\left\|\widehat{\Phi}_{1}\right\|_{H}^{2}, \\
& \left\|\widehat{\Phi}_{0}\right\|_{V}^{2}+\left\|\widehat{\Phi}_{1}\right\|_{H}^{2} \leq C_{2} \int_{0}^{T}\left(\left|\widehat{\phi}_{t}(L, t)-\widehat{\phi}_{t}(L, 0)\right|^{2}+\widehat{\phi}_{x t t}^{2}(L, t)\right) d t .
\end{aligned}
$$

On the other hand, since

$$
\widehat{\Phi}_{t t}(0)=-A \widehat{\Phi}(0)=\Phi_{0}, \quad \widehat{\Phi}_{t t t}(0)=-A \widehat{\Phi}_{t}(0)=\Phi_{1}
$$

then we have $\widehat{\Phi}_{t t}=\Phi$. It follows that

$$
\widehat{\phi}_{x t t}(L, t)=\phi_{x}(L, t), \quad \widehat{\phi}_{t}(L, t)-\widehat{\phi}_{t}(L, 0)=\int_{0}^{t} \phi(L, s) d s .
$$

Inserting $(4.7)$ into $(4.5,4.6)$ and using $(4.3)$, we obtain the inequalities $(4.1,4.2)$. The proof is complete. 
Theorem 4.2. Let $\Phi(x, t)=\left(\phi(x, t), \phi(L, t), \phi_{x}(L, t)\right)$ be the solution of the equation (2.11). Assume that $L<3$. Then for any $T>0$, there exists constant $C_{2}>0$ depending only on $L, T$ such that the following estimate holds

$$
\left\|\Phi_{0}\right\|_{V^{\prime}}^{2}+\left\|\Phi_{1}\right\|_{D(A)^{\prime}}^{2} \leq C_{2} \int_{0}^{T} \phi_{x}^{2}(L, t) d t
$$

Proof. The proof is similar to that of Theorem 4.1. It is sufficient to apply the inequality (2.42) to the solution $\widehat{\Phi}$ of the equation (4.4).

Now we consider the exact controllability of the equation (3.11) by means of regular controls

$$
v_{0}=-\int_{T}^{t} \widetilde{v}_{0}(s) d s \in H^{1}(0, T), \quad v_{1}(t) \in L^{2}(0, T) .
$$

For the wellposedness of the equation (3.11) with the controls (4.9), we first interpret (3.14) into the following form

$$
\mathcal{L}\left(\Phi_{0}, \Phi_{1}\right)=\left\langle\left(-u_{t}(t), u(t)\right), S(t)\left(\Phi_{0}, \Phi_{1}\right)\right\rangle_{V \times D(A), V^{\prime} \times D(A)^{\prime}}
$$

where the linear form $\mathcal{L}$ is defined by

$$
\mathcal{L}\left(\Phi_{0}, \Phi_{1}\right)=\int_{0}^{t} \widetilde{v}_{0}(s)\left(\int_{0}^{s} \phi(L, \tau) d \tau\right) d s+\int_{0}^{t} v_{1}(s) \phi_{x}(L, s) d s+\left\langle u_{0}, \Phi_{1}\right\rangle_{D(A) \times D(A)^{\prime}}-\left\langle u_{1}, \Phi_{0}\right\rangle_{V \times V^{\prime}}
$$

Theorem 4.3. Let $v_{0}, v_{1}$ be chosen in (4.9). Then for any $\left(u_{0}, u_{1}\right) \in D(A) \times V$ and $\left(v_{0}, v_{1}\right) \in H^{1}(0, T)$ $\times L^{2}(0, T)$, the equation (3.11) admits a unique weak solution $u$ such that

$$
u \in C^{0}([0, T] ; D(A)) \cap C^{1}([0, T] ; V)
$$

defined in the sense that the equation (4.10) is satisfied for all $0<t<T$ and all $\left(\Phi_{0}, \Phi_{1}\right) \in V^{\prime} \times D(A)^{\prime}$. Moreover the linear application

$$
\left(u_{0}, u_{1}, v_{0}, v_{1}\right) \rightarrow\left(u, u_{t}\right)
$$

is continuous for the corresponding topologies.

Proof. First using the direct inequality (4.1), a straightforward computation gives that

$$
\begin{aligned}
\mid \int_{0}^{t}\left(\widetilde{v}_{0}(s) \int_{0}^{s} \phi(L, \tau) d \tau\right) d s & +\left.\int_{0}^{t} v_{1}(s) \phi_{x}(L, s) d s\right|^{2} \\
& \leq\left(\left\|\widehat{v}_{0}\right\|_{L^{2}(0, T)}^{2}+\left\|\widetilde{v}_{1}\right\|_{L^{2}(0, T)}^{2}\right) \int_{0}^{T}\left(\int_{0}^{s}|\phi(L, \tau)|^{2} d \tau+\left|\phi_{x}(L, s)\right|^{2}\right) d s \\
& \leq C\left(\left\|v_{0}\right\|_{H^{1}(0, T)}+\left\|v_{1}\right\|_{L^{2}(0,1)}\right)\left(\left\|\Phi_{0}\right\|_{V^{\prime}}+\left\|\Phi_{1}\right\|_{D(A)^{\prime}}\right)
\end{aligned}
$$

This implies that the linear form $\mathcal{L}$ is continuous in the space $V^{\prime} \times D(A)^{\prime}$. Moreover we have

$$
\|\mathcal{L}\| \leq C\left(\left\|u_{0}\right\|_{D(A)}+\left\|u_{1}\right\|_{V}+\left\|v_{0}\right\|_{H^{1}(0, T)}+\left\|v_{1}\right\|_{L^{(0,1)}}\right) .
$$


From Riesz's representation theorem, there exists a unique $\left(U(t), U_{t}(t)\right) \in D(A) \times V$ such that

$$
\mathcal{L}\left(\Phi_{0}, \Phi_{1}\right)=\left\langle\left(-U_{t}(t), U(t)\right),\left(\Phi_{0}, \Phi_{1}\right)\right\rangle_{H \times V, H \times V^{\prime}}
$$

for all $\left(\Phi_{0}, \Phi_{1}\right) \in H \times V^{\prime}$. Then indeed setting

$$
\left(-u_{t}(t), u(t)\right):=S(t)\left(-U_{t}(t), U(t)\right)
$$

we get the equation (4.10). Moreover we have

$$
\|u(t)\|_{D(A)}+\left\|u_{t}(t)\right\|_{V} \leq C\left(\left\|u_{0}\right\|_{D(A)}+\left\|u_{1}\right\|_{V}+\left\|v_{0}\right\|_{H^{1}(0, T)}+\left\|v_{1}\right\|_{L^{(0,1)}}\right) .
$$

Using the same argument as that at the end of the proof of Theorem 3.1, we prove the regularity (4.11) of the weak solution and the continuous dependence of the application (4.13).

Theorem 4.3. Let $T>0$. Then for all $\left(u_{0}, u_{1}\right) \in D(A) \times V$, there exist two controls $v_{0} \in H^{1}(0, T)$ and $v_{1} \in L^{2}(0, T)$ such that the weak solution $u$ of the problem (3.11) satisfies the final conditions (3.21).

Proof. Let $\Phi$ be the weak solution of the homogeneous equation $(2.11)$ with the initial data $\left(\Phi_{0}, \Phi_{1}\right) \in V^{\prime} \times D(A)^{\prime}$. Defining the semi-norm

$$
\left\|\left(\Phi_{0}, \Phi_{1}\right)\right\|_{F}^{2}=\int_{0}^{T}\left\{\left(\int_{0}^{t} \phi(L, s) d s\right)^{2}+\phi_{x}^{2}(L, t)\right\} d t
$$

then thanks to the inequalities $(4.1,4.2)$ we know that (4.17) defines an equivalent norm in the space $F=V^{\prime} \times D(A)^{\prime}$.

Taking the controllers $v_{0}, v_{1}$ as follows

$$
v_{0}(t)=-\int_{T}^{t} \int_{0}^{s} \phi(L, \tau) d \tau d s, \quad v_{1}(t)=\phi_{x}(L, t)
$$

from the direct inequality (4.1), we have

$$
\left\|v_{0}\right\|_{H^{1}(0, T)}^{2}+\left\|v_{1}\right\|_{L^{2}(0, T)}^{2} \leq C\left(\left\|\Phi_{0}\right\|_{V^{\prime}}^{2}+\left\|\Phi_{1}\right\|_{D(A)^{\prime}}^{2}\right) .
$$

Next we solve the backward problem (3.25) with the right-hand side given by (4.18), and define the operator $\Lambda$ as in $(3.25)$. By virtue of $(4.12,4.16)$ and $(4.19)$ we see that $\Lambda$ is a linear continuous operator from $V^{\prime} \times D(A)^{\prime}$ into $D(A) \times V^{\prime}$.

Now multiplying the equation (3.25) by $\Phi$ and integrating by parts, we obtain that

$$
-\left(u_{0}, \Phi_{1}\right)_{H}+\left(u_{1}, \Phi_{0}\right)_{H}=\int_{0}^{T}\left\{\left(\int_{0}^{t} \phi(L, s) d s\right)^{2}+\phi_{x}^{2}(L, t)\right\} d t .
$$

Interpreting (4.20) into the following form

$$
\left\langle\Lambda\left(\Phi_{0}, \Phi_{1}\right),\left(\Phi_{0}, \Phi_{1}\right)\right\rangle_{V \times D(A), V^{\prime} \times D(A)^{\prime}}=\left\|\left(\Phi_{0}, \Phi_{1}\right)\right\|_{F}^{2}
$$

and using Lax-Milgram's theorem we deduce that $\Lambda$ is an isomorphism from $V^{\prime} \times D(A)^{\prime}$ onto $V \times D(A)$. Therefore given any $\left(u_{1},-u_{0}\right) \in H \times V$, there exists a unique $\left(\Phi_{0}, \Phi_{1}\right) \in H \times V^{\prime}$ such that

$$
\Lambda\left(\Phi_{0}, \Phi_{1}\right)=\left(u_{t}(0),-u(0)\right)=\left(u_{1},-u_{0}\right) .
$$


This means precisely that the weak solution $u$ of the backward problem (3.25), with the right-hand side $v$ given by (4.18), satisfies the initial value conditions (3.31). On other word, we have proved that the system (3.11) is driven to rest by the singular controls $v_{0}, v_{1}$ given in (4.18). The proof is thus complete.

Theorem 4.4. Let $L<3$ and $T>0$. Then for any $\left(u_{0}, u_{1}\right) \in D(A) \times V$ there exists a controller $v_{1} \in L^{2}(0, T)$ such that the weak solution $u$ of the controlled problem (3.11) satisfies the final conditions (3.21).

Proof. Let $\Phi(t)$ be the solution of the homogeneous equation (2.11). We define the semi-norm

$$
\left\|\left(\Phi_{0}, \Phi_{1}\right)\right\|_{F}^{2}=\int_{0}^{T}\left|\phi_{x t}(L, t)\right|^{2} d t .
$$

Thanks to the inequalities (4.1) and (4.8), we know that (4.21) defines an equivalent norm in the space $F=V^{\prime} \times D(A)^{\prime}$.

We next solve the the backward problem (3.25) with the following controls

$$
v_{0}=0, \quad v_{1}(t)=\phi_{x}(L, t) \in L^{2}(0, T) .
$$

Defining the operator $\Lambda$ as in (3.25), then by virtue of (4.16) and (4.19) we see that $\Lambda$ is a linear continuous operator from $V^{\prime} \times D(A)^{\prime}$ into $V \times D(A)$.

Multiplying the equation (3.25) by $\Phi$ and integrating by parts so that we obtain that

$$
\left\langle\Lambda\left(\Phi_{0}, \Phi_{1}\right),\left(\Phi_{0}, \Phi_{1}\right)\right\rangle_{V \times D(A), V^{\prime} \times D(A)^{\prime}}=\left\|\left(\Phi_{0}, \Phi_{1}\right)\right\|_{F}^{2} .
$$

Therefore $\Lambda$ is an isomorphism from $V^{\prime} \times D(A)^{\prime}$ onto $D(A) \times V$. Using the principle of the HUM, we conclude that for any $\left(u_{1},-u_{0}\right) \in V \times D(A)$ there exists a controller $v_{1} \in L^{2}(0, T)$ such that the weak solution $u$ of the backward problem (3.11) satisfies the final conditions (3.21). The proof is thus complete.

\section{REFERENCES}

[1] C. Castro and E. Zuazua, Boundary controllability of a hybrid system consisting in two flexible beams connected by a point mass. SIAM J. Control Optim. 36 (1998) 1576-1595.

[2] S. Hanssen and E. Zuazua, Exact controllability and stabilization of a vibration string with an interior point mass. SIAM $J$. Control Optim. 33 (1995) 1357-1391.

[3] W. Littman and L. Markus, Exact boundary controllability of a hybrid system of elasticity. Arch. Rational Mech. Anal. 103 (1988) 193-236.

[4] W. Littman and L. Markus, Stabilization of a hybrid system of elasticity by feedback boundary damping. Ann. Mat. Pura Appl. 152 (1988) 281-330.

[5] J.L. Lions, Contrôlabilité exacte, perturbations et stabilisation de systèmes distribués, Vol. I. Masson, Paris (1988).

[6] J.L. Lions, Exact controllability, stabilizability, and perturbations for distributed systems. SIAM Rev. 30 (1988) 1-68.

[7] L. Markus and Y.C. You, Dynamical boundary control for elastic Al plates of general shape. SIAM J. Control Optim. 31 (1993) 983-992.

[8] S. Micu and E. Zuazua, Boundary controllability of a linear hybrid system arising in the control noise. SIAM J. Control Optim. 35 (1987) 1614-1637.

[9] A. Pazy, Semigroups of linear operators and applications to partial differential equations. Springer-Verlag, New York (1983).

[10] B. Rao, Stabilisation du modèle SCOLE par un contrôle frontière a priori borné. C. R. Acad. Sci. Paris Sér. I Math. 316 (1993) 1061-1066.

[11] B. Rao, Uniform stabilization and exact controllability of Kirchhoff plates with dynamical boundary controls.

[12] B. Rao, Uniform stabilization of a hybrid system of elasticity. SIAM J. Control Optim. 33 (1995) 440-454.

[13] B. Rao, Contrôlabilité exacte frontière d'un système hybride en élasticité par la méthode HUM. C. R. Acad. Sci. Paris Sér. I Math. 324 (1997) 889-894.

[14] J. Simon, Compact sets in the space $L^{p}(0, T ; B)$. Ann. Mat. Pura Appl. (IV) CXLVI (1987) 65-96.

[15] M. Slemrod, Feedback stabilization of a linear system in Hilbert space with an a priori bounded control. Math. Control Signals Systems (1989) 265-285.

[16] E. Zuazua, Contrôlabilité exacte en un temps arbitrairement petit de quelques modèles de plaques, in Lions [5], 465-491. 